\title{
Bilayered skin-substitute technology for the treatment of diabetic foot ulcers: current insights
}

This article was published in the following Dove Press journal:

Chronic Wound Care Management and Research

21 December 2016

Number of times this article has been viewed

Jeffrey C Karr

Karr Foot and Leg Center, Lakeland, FL, USA
Correspondence: Jeffrey C Karr

Karr Foot and Leg Center, 542I South

Florida Avenue, Lakeland, FL 33813, USA

$\mathrm{Tel}+\mathrm{I} 8636465960$

Fax + I 8636442847

Email drkarrcoe@gmail.com
Abstract: The estimated prevalence of diabetes mellitus in New Zealand is 7\%, and as in many other developed countries is a growing problem. One of the most common and costly complications, diabetic foot ulcers (DFUs) are chronic wounds that result when the phases of wound healing are disrupted or incomplete, resulting in wounds that persist for several months or even years. Despite standard-care therapy (ie, debridement, infection elimination, use of dressings, off-loading), the majority of DFUs remain unhealed, and it is thus appropriate to consider advanced therapies. One such therapy is a bioengineered bilayered living cellular construct (BLCC) comprised of living keratinocytes and fibroblasts. BLCC facilitates the delivery of a broad array of cytokines and growth factors often deficient in chronic nonhealing wounds, and in doing so reverses patients' wounds from a chronic wound to an acute normally healing wound. BLCC has an important body of evidence to support its use in DFUs, including randomized clinical trials, a real-world comparative-effectiveness analysis, and health-economics data. Keywords: bilayer skin substitute, bilayered living cellular construct, chronic wounds, diabetic foot ulcers, wound healing

\section{Introduction}

Based on the New Zealand's Medicines Landscape: 2015 report, more than 257,000 New Zealanders have diabetes mellitus, and the number of people with diabetes grows by nearly 40 people each day. ${ }^{1}$ In a cross-sectional survey based on the 2008-2009 New Zealand Adult Nutrition Survey, the prevalence of diabetes was $7 \% .^{2}$ Diabetes is most common among Maori and Pacific Islanders: Maori are three times more likely to develop type 2 diabetes than non-Maori; they are also five times more likely to die from it. In Australia, diabetes resulted in over 500,000 hospital admissions (representing $8 \%$ of all admissions) and 12,000 deaths (representing 9\% of all deaths) in 2004 alone. $^{3}$ Diabetes contributes significantly to cardiovascular disease, and is the leading cause of kidney failure, blindness (in people aged under 60 years), and lowerlimb amputations. Recent reports suggest that Australia has one of the worst rates of diabetes-related lower-limb amputations in a developed country, at nearly 20 per 100,000 people with diabetes. ${ }^{4}$

Diabetic foot ulcers (DFUs) are chronic wounds that are common and costly complications of diabetes. ${ }^{5}$ In Australia, DFUs result in longer average length of hospital stay compared to all other diabetes-related complications (including cardiovascular disease and kidney disease) and can cost an estimated $\$ 16,700$ per person annually. ${ }^{4}$ Patients with diabetes mellitus have a $15 \%$ lifetime risk of developing an DFU. ${ }^{6,7}$ Multiple risk factors contribute to developing DFUs, including diabetic 
peripheral neuropathy, peripheral vascular disease, poor glycemic control, foot deformity, high plantar pressure, infections, and inappropriate foot self-care habits. To date, most DFUs are caused by neuropathic, ischemic, or combined neuroischemic abnormalities. ${ }^{5}$ Standard management for DFUs consists of debridement, infection elimination, use of dressings, and off-loading. However, the majority of DFUs do not respond to standard care and remain unhealed.

For chronic, nonhealing ulcers, advanced therapies, such as skin-replacement therapies, should be considered, and their use is supported by the Wound Healing Society (WHS) published guidelines for the treatment of DFUs. ${ }^{8}$ One such therapy is a bilayered living cellular construct (BLCC; Apligraf; Organogenesis Inc, Canton, MA, USA), a bioengineered skin-substitute technology approved for the treatment of nonhealing DFUs and venous leg ulcers (VLUs). A BLCC is comprised of human neonatal keratinocytes and fibroblasts in an extracellular matrix (ECM) of bovine and human collagen and other ECM proteins. It was the first allogeneic cell-based product approved by the US Food and Drug Administration (FDA). The product was approved as a class III premarket approval device, which requires the most stringent FDA review, including randomized controlled clinical trial data to support each indication. The purpose of this article is to review the normal wound-healing process; our current understanding of the underlying mechanisms that result in the "stalled" or nonhealing chronic nature of DFUs; current management guidelines for DFUs; and the role of an evidence base supporting the use of advanced therapy with a BLCC for the treatment of DFUs. The BLCC is currently the only bilayered skin-substitute technology available on the market, and is the focus of this review.

\section{The normal wound-healing process}

Wound healing is a complex, highly regulated process involving three overlapping series of events to restore tissue integrity and homeostasis: inflammation, proliferation, and remodeling. ${ }^{9}$ Along this continuum, multiple cell types (eg, fibroblasts, keratinocytes), signaling molecules (ie, cytokines and growth factors critical to coordinating cellular processes), and proteases (involved in ECM deposition and remodeling) are activated. ${ }^{10}$ Cytokines and growth factors are small polypeptides secreted by different cell types to modulate and coordinate cellular proliferation, differentiation, migration, and metabolism. Different cytokines and growth factors are upregulated depending on the phase of wound healing, and in fact studies have found reduced concentrations of these molecules in nonhealing wounds compared with acute healing wounds. ${ }^{11}$
Upon acute injury to the skin, platelets activate the formation of a fibrin clot, which serves as a provisional wound matrix for cell migration. Immune cells predominate during the inflammatory phase to remove bacteria and nonviable tissue from the wound bed. Numerous cytokines and growth factors are released to recruit fibroblasts, endothelial cells, and keratinocytes to stabilize the wound and to repair the damaged blood vessels. The inflammatory phase may last from a few days to weeks, and as it subsides with the apoptosis of immune cells, the proliferative phase begins. ${ }^{12}$ The proliferative phase is characterized by angiogenesis, tissue granulation, and reepithelialization. Signaling molecules trigger new blood vessels to form, and fibroblasts proliferate to form the ECM. Keratinocytes are then activated and migrate over the injured dermis to cover the wound surface with a layer of epithelium. After epithelial wound closure has been achieved, the remodeling phase begins. The remodeling phase can continue for 6 months to a year or longer. During this time, collagen synthesis and degradation work to reestablish an equilibrium that results in a stable, mature scar. ${ }^{13}$ At the end of the wound-healing process, the wound is completely closed; however, the repaired tissue never fully regains the properties of the original tissue structure. ${ }^{9,11}$

\section{Pathophysiology of wound healing in DFUs}

In practical terms, wounds are described as acute wounds if they follow the phases of wound healing, ultimately healing in a matter of several weeks. Chronic, nonhealing wounds, such as DFUs, result when the phases of wound healing are disrupted or incomplete, resulting in wounds that persist for several months or even years. ${ }^{11,14}$ When wounds fail to heal, the goal of treatment is to convert the molecular and cellular environment of a chronic wound bed into that of an acute healing wound, such that the healing can proceed through the normal wound-healing process. ${ }^{15}$

DFUs readily become chronic wounds as a result of many pathophysiologic defects that cause them to be "stuck" in either the inflammatory or proliferative phases. On a clinical level, peripheral neuropathy can result in the loss of protective sensation to the foot, such that the injury goes unnoticed. Arterial insufficiency can further complicate the neuropathic ulcer and impair the healing process. ${ }^{16}$ Foot deformity, calluses, and mechanical stress at the wound site have all been implicated in affecting wound healing. ${ }^{17}$ Uncontrolled hyperglycemia adversely affects the wound-healing process by promoting infection and altering cellular function. ${ }^{5}$ Optimizing glucose control in patients with diabetes is thus 
essential, not only as part of good diabetes management but also to improve wound healing in DFUs.

Patients with diabetes may also have an altered immune function contributing to poor wound healing. Studies have shown decreased chemotaxis, phagocytosis, and bacterial killing in the inflammatory phase of wound healing in diabetes. ${ }^{16,18}$ Chronic wounds often have a prolonged inflammatory phase as a result of bacterial bioburden. ${ }^{13,19}$ All chronic wounds contain a bacterial bioburden that can range from free-floating, planktonic microorganisms to a more complex surface-attached clusters of bacteria encased within a protective extracellular polymeric substance (Figure 1). These communities of bacteria, defined as biofilms, differ from their planktonic equivalents in that they show high resistance to antibiotic therapy. Biofilms are implicated in the inability of many wounds to heal, and their formation is extremely common in chronic wounds. An estimated $60 \%-90 \%$ of chronic wounds contain biofilm compared with only $6 \%$ of acute wounds. ${ }^{8,20-22}$ The necrotic tissue and debris help to facilitate bacterial attachment, and an impaired host immune response helps to foster bacterial growth. ${ }^{23}$ Sharp surgical debridement is an effective method to remove biofilm. However, even with debridement, biofilm reforms quickly, and it is thus important to follow up debridement with appropriate antibiotics and wound-management products. ${ }^{24}$

Wound healing in patients with diabetes is also impaired as a result of dysfunctional wound cells and imbalances in key cytokines, growth factors, and proteases. Many DFUs appear to stall in the proliferative phase. Cells in chronic wounds, including fibroblasts and keratinocytes, are often morphologically and functionally abnormal. In addition, there is decreased proliferation and abnormal differentiation. ${ }^{25}$ There is an increased number of fibroblasts in the nonproliferative state. Further, there appears to be an altered pattern of cytokine appearance and abnormal expression of growth factors in DFUs, resulting in impaired communication and functioning between cell types. Consequently, fibroblasts have been found to have an impaired ability to remodel the ECM, and keratinocytes may not function adequately to reepithelialize the wound. ${ }^{26}$ Hyperglycemia alters the balance of expression of matrix metalloproteinases (MMPs) and tissue inhibitors of metalloproteinases (TIMPs),

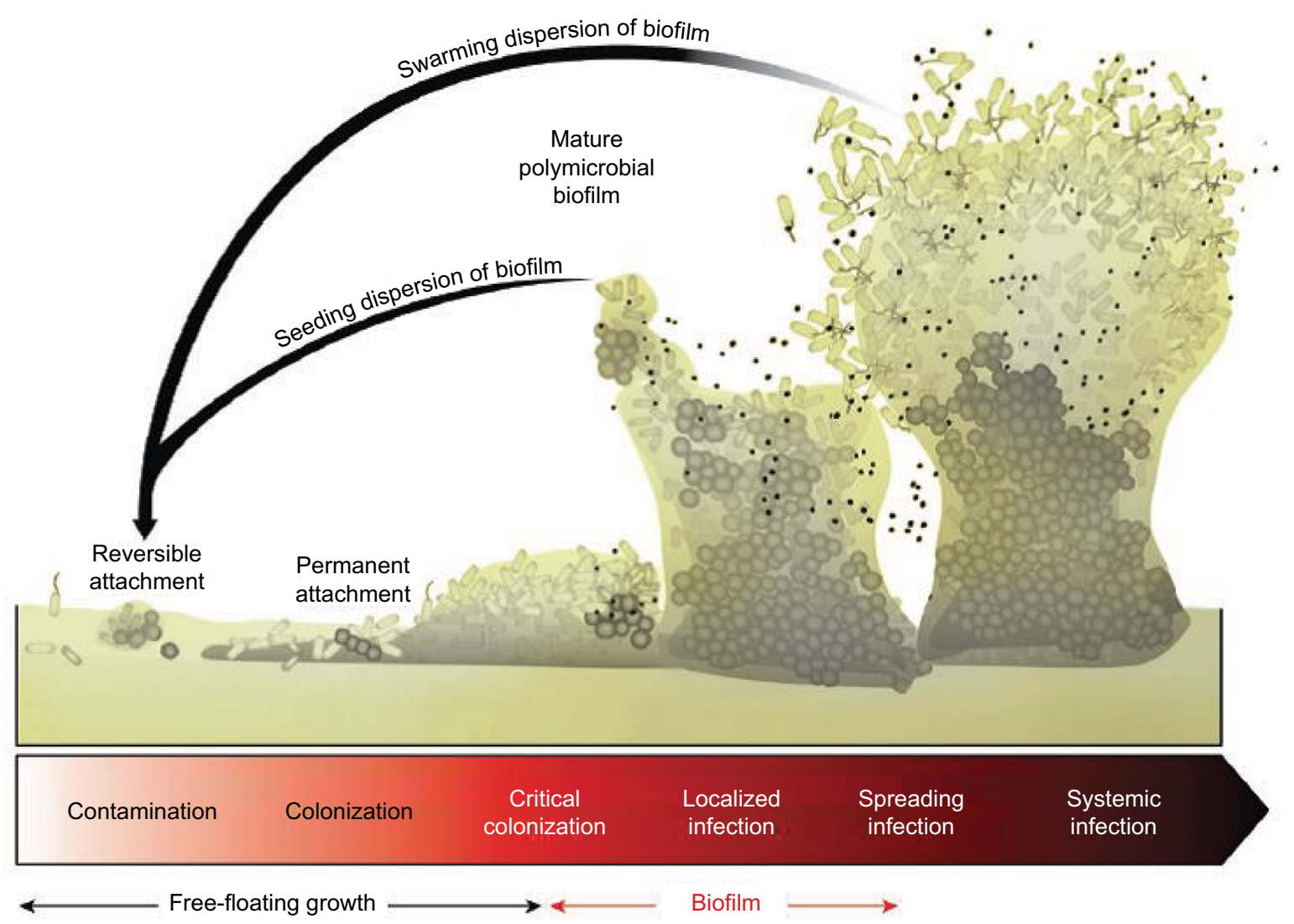

Requires therapeutic intervention

Figure I Schematic representation of polymicrobial biofilm formation.

Notes: Phillips P, Sampson E, Yang Q, Antonelli P, Progulske-Fox A, Schultz G. Bacterial biofilms in wounds. Wound Heal S Afr. 2008; I (2): I0-I2. ${ }^{20}$ 
ie, it specifically increases levels of MMPs and reduces levels of TIMPs, which results in degradation of the ECM. ${ }^{16,27}$ MMPs are implicated in degrading growth factors and their target-cell receptors, preventing healing. ${ }^{15}$

\section{Standard wound-care management in DFUs}

Standard treatment for DFUs includes debridement, infection control, use of moisture dressings, and off-loading high pressure. ${ }^{28}$ Debridement to remove necrotic tissue, excessive bacterial burden, and dead and senescent cells is a critical component of preparing and maintaining the wound bed, and is widely known to improve the healing potential of the wound. Sharp surgical debridement is the most effective and preferred method of debridement. If there is suspected infection in a debrided wound, cultures should be obtained to determine the wound pathogens, and the appropriate antibiotic treatment should be prescribed. In granulating wounds, topically applied antimicrobials should be used to decrease bacterial levels. Oral or parenteral antibiotics may be needed for more severe infections. There are numerous dressings for the management of DFUs. The goal of these dressings are to maintain a moist wound-healing environment. The clinician should consider the cost, ease of use, and potential for iatrogenic injury and wound-exudate management when selecting a dressing approach. Clinical trials have not yet shown that any one dressing approach is better than another to facilitate the wound-healing process. The use of off-loading techniques is considered one of the most important components of managing DFUs. Many studies provide evidence showing that by reducing pressure and preventing repetitive trauma to the sole of the foot, proper off-loading helps to promote DFU healing and can prevent recurrent ulcers. ${ }^{28}$ Unfortunately, standard wound care alone is often unsuccessful in completely healing DFUs. It is estimated that approximately $70 \%$ of DFUs do not heal by 20 weeks with standard-care therapy. ${ }^{29}$

A thorough assessment of the patient and the wound is critical at the initial visit, and patients should be followed up weekly. ${ }^{8}$ The WHS guidelines recommend ongoing and consistent documentation of wound history, recurrence, and characteristics (including location, size, base, exudates, condition of the surrounding skin, staging, and pain) to evaluate wound-bed preparation. ${ }^{28}$ Obtaining a precise baseline measure of wound size is particularly important, in order to assess response to treatment. If the ulcer is not healing at the expected rate, then the interventions for wound-bed preparation should be reassessed. ${ }^{8,28}$ Based on the evidence, ${ }^{30-32}$ the WHS treatment guidelines recommend a change in treatment course if wound-size reduction of at least $50 \%$ is not observed after 4 weeks of standard care. ${ }^{28}$ In the absence of underlying disease or patient nonadherence to prescribed standard treatment (eg, insufficient off-loading), intervention at this point with advanced therapies, such as BLCC, in combination with standard care is appropriate and consistent with typical treatment algorithms (Figure 2). The presence of negative predictors for DFU healing also can aid in identifying a nonhealing wound and the potential need to intervene earlier with advanced therapies. For example, larger wounds and longer wound durations are associated with a decreased likelihood of healing. One study found that only $26 \%$ of ulcers $>4 \mathrm{~cm}^{2}$ and $>12$-month duration healed compared with $43 \%$ of ulcers $<2 \mathrm{~cm}^{2}$ and $<6$-month duration. ${ }^{33}$ Other negative predictors include the presence or extent of arterial insufficiency, the degree of neuropathy, ulcer location, presence of Charcot deformity, and the propensity for infection..$^{27}$ Early intervention with advanced treatment expedites wound healing and leads to better patient care. ${ }^{34,35}$

\section{Advanced therapy with BLCC: review of the evidence}

BLCC is an advanced wound care therapy bioengineered to mimic the structure and function of human skin. It is a living, bilayered (ie, dermal and epidermal layer) technology that contains two cell types relevant to healing. The upper epidermal layer contains human keratinocytes that multiply and differentiate to form a stratum corneum barrier, and the basal layer contains keratinocyte stem cells (Figure 3). The keratinocyte stem cells in BLCC are essential to form its differentiated epidermis. In vitro data show that without the keratinocyte stem cells, epidermis formation is significantly impaired..$^{36}$ The lower layer of the BLCC contains human fibroblasts in an ECM.

The presence of both fibroblasts and keratinocytes in BLCC has been shown in vitro to facilitate the delivery of a broad array of cytokines and growth factors that are often deficient in chronic wounds but integral for normal wound healing (Table 1). The number of signaling molecules produced is actually fewer if only one cell type is present. ${ }^{37,38}$ In addition, the ECM in BLCC contains TIMPs, which are potent inhibitors of MMPs. ${ }^{39}$ Increased levels of TIMPs are important to improve the MMP imbalance in chronic wounds. Unlike human skin, BLCC does not contain melanocytes, macrophages, lymphocytes, Langerhans cells, blood vessels, hair follicles, or sweat glands. BLCC does not engraft to the patient, ie, there is no vascularization or integration of BLCC into the wound. ${ }^{40,41}$ Rather, BLCC serves as a temporary 


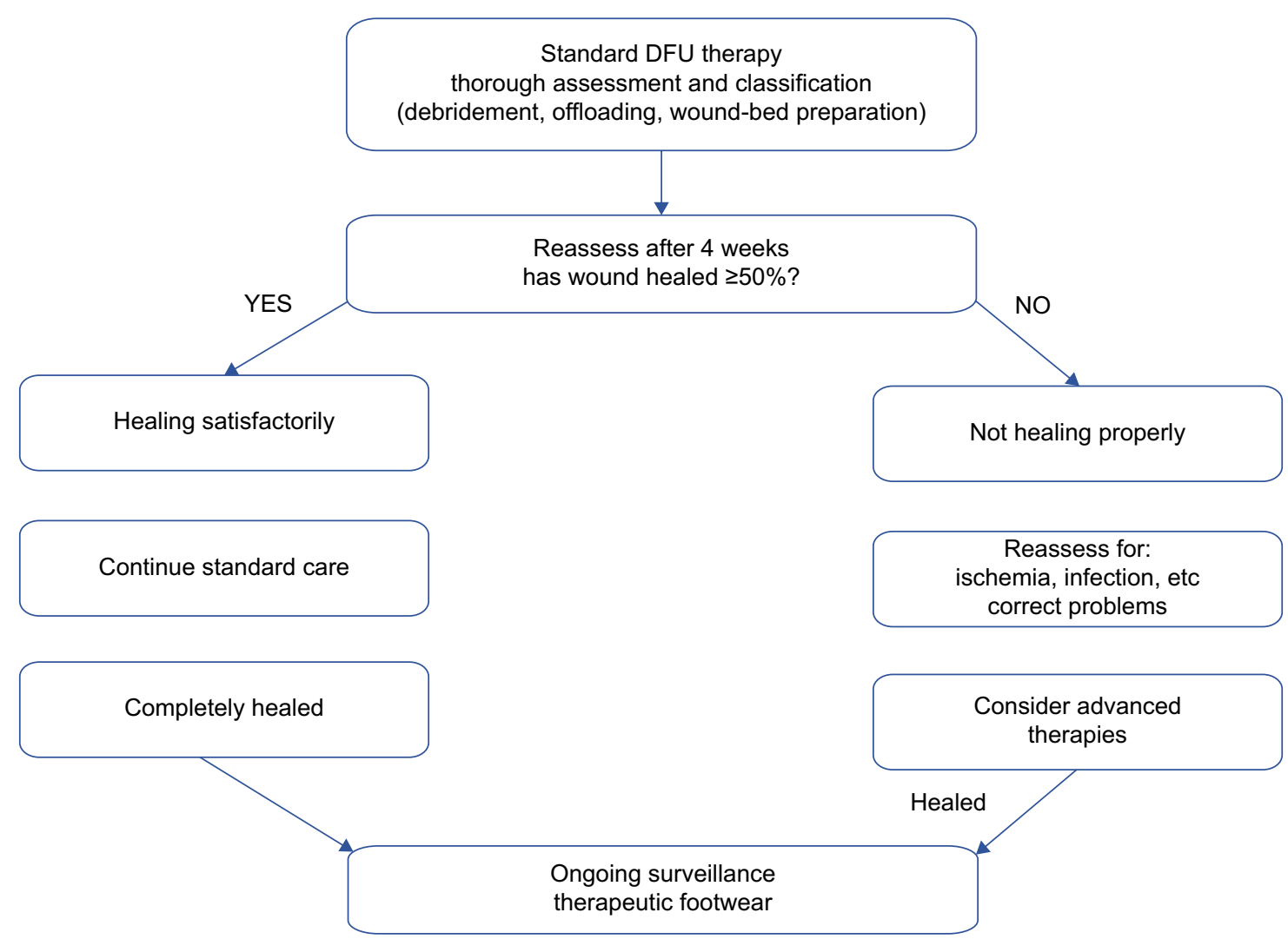

Figure 2 Simplified treatment algorithm for DFU treatment.

Notes: The publisher for this copyrighted material is Mary Ann Liebert, Inc. publishers. Copyright @20I5. Mary Ann Liebert, Inc. Reproduced from Frykberg RG, Banks J. Challenges in the treatment of chronic wounds. Adv Wound Care. 2015;4(9):560-582. ${ }^{13}$

Abbreviation: DFU, diabetic foot ulcer.
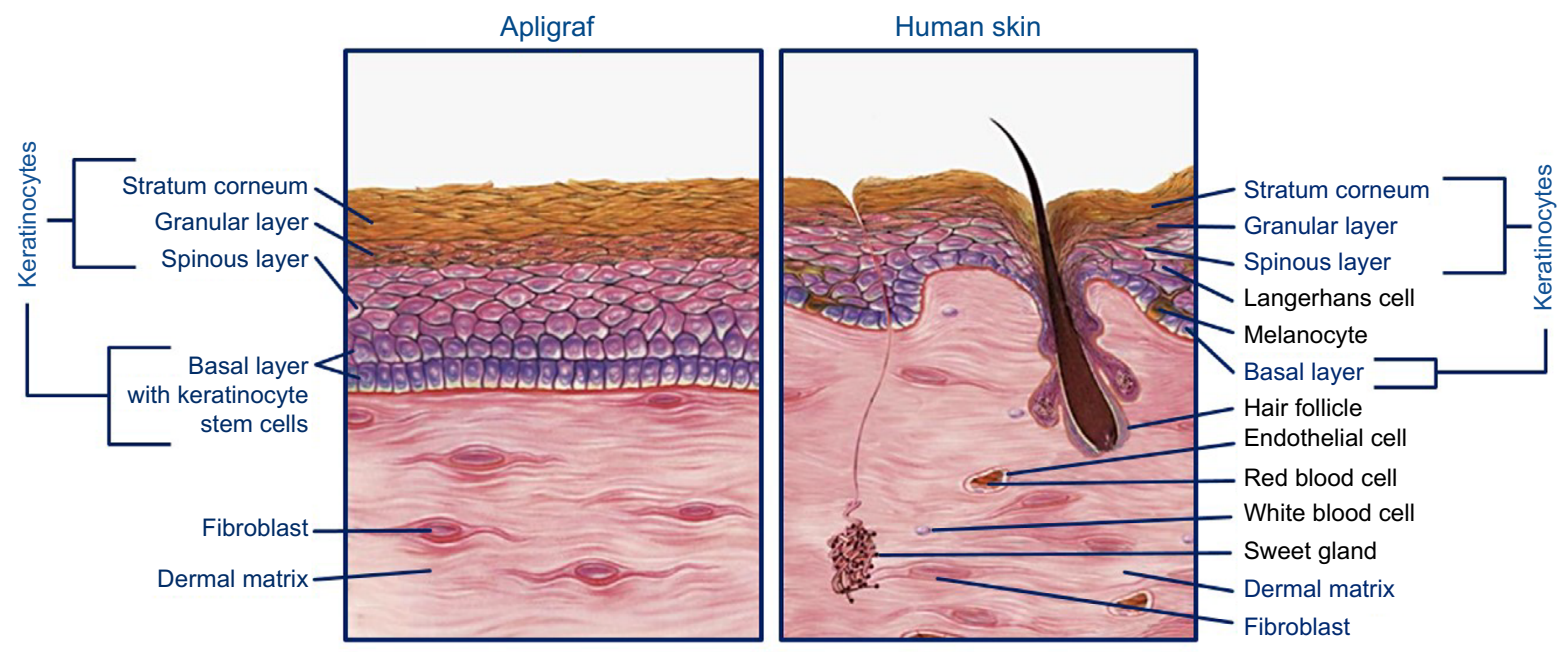

Figure $3 \mathrm{BLCC}$ is a bioengineered, living cellular technology with two cell types relevant to healing (illustration courtesy of Organogenesis Inc). Abbreviation: BLCC, bilayered living cellular construct.

barrier function during healing, and by delivering a broad array of signaling molecules, BLCC helps to accelerate the wound-healing process.

A recent study out of the University of Miami evaluated the changes in patients' healing profiles following BLCC application. ${ }^{42}$ These were patients with non-healing VLUs with less than $40 \%$ area reduction after 4 weeks of conventional therapy. Biopsies were obtained from both the base and the edge of unresponsive VLUs to assess the baseline profile of the chronic wound. Patients were then randomized to BLCC plus conventional therapy or conventional therapy only. At the end of 1 week, biopsies were performed again to evaluate changes in the profile from baseline to day 7. Investigators then compared the new biopsies with biopsies taken of acute healing wounds that were available in their archives. Results showed that BLCC reversed the inflammatory 
Table I BLCC produces a broad spectrum of cytokines and growth factors through the combined effect of keratinocytes and fibroblasts

\begin{tabular}{|c|c|c|c|c|}
\hline Function & Growth factor/cytokine ${ }^{a}$ & Human keratinocytes $^{b}$ & Human fibroblasts ${ }^{b}$ & BLCC \\
\hline \multirow[t]{3}{*}{ Angiogenesis } & PDGFA $^{c}$ & $\checkmark$ & $\checkmark$ & $\checkmark$ \\
\hline & $\mathrm{PDGFB}^{\mathrm{c}}$ & $\checkmark$ & $\checkmark$ & $\checkmark$ \\
\hline & VEGFc & $\checkmark$ & $\checkmark$ & $\checkmark$ \\
\hline Growth, development, & IGFI & & & $\checkmark$ \\
\hline \multirow[t]{3}{*}{ and differentiation } & IGF2 & & $\checkmark$ & $\checkmark$ \\
\hline & TGF $\beta_{1}$ & & $\checkmark$ & $\checkmark$ \\
\hline & $\mathrm{TGF}_{3}$ & & $\checkmark$ & $\checkmark$ \\
\hline \multirow[t]{4}{*}{ Inflammation } & $\mathrm{IL}-\mathrm{I} \alpha^{\mathrm{c}}$ & $\checkmark$ & & $\checkmark$ \\
\hline & IL-6c & & $\checkmark$ & $\checkmark$ \\
\hline & IL-8c & $\checkmark$ & $\checkmark$ & $\checkmark$ \\
\hline & $I L-I^{c}$ & & $\checkmark$ & $\checkmark$ \\
\hline Proliferation & TGF $\alpha^{c}$ & $\checkmark$ & & $\checkmark$ \\
\hline
\end{tabular}

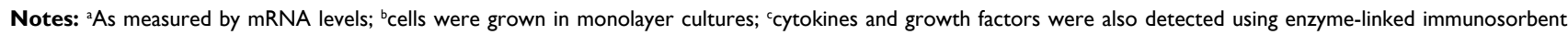
assay. Data from Barrientos et $\mathrm{al}^{25}$ and Brem et al. ${ }^{34}$

Abbreviations: BLCC, bilayered living cellular construct.

environment, activated keratinocytes at the wound edge, balanced ECM production and remodeling of the wound base, and regulated and corrected growth-factor signaling. The investigators found that BLCC actually reversed patients' wounds from a chronic profile to an acute profile.

\section{Randomized controlled trials}

The pivotal trial of BLCC in DFUs was a prospective, randomized controlled clinical trial that recruited patients from 24 US centers. ${ }^{43}$ Key inclusion criteria included type 1 or 2 diabetes, age 18 to 80 years, $A_{1 c}$ between $6 \%$ and $12 \%$, and full-thickness neuropathic ulcers. Ulcers were present on the plantar, medial, or lateral surfaces of the foot for $\geq 3$-week duration. Ulcer size postdebridement had to be $1-16 \mathrm{~cm}^{2}$. A total of 208 patients were randomized to treatment with BLCC or conventional therapy with saline-moistened gauze (active control). Both groups also received standard state-ofthe-art adjunctive therapy, which included extensive surgical debridement and adequate foot off-loading. The primary end point was complete wound closure, defined as full epithelialization of the wound with the absence of drainage, from baseline to week 12 . The study found that significantly more BLCC-treated patients achieved complete wound closure compared with the control group at 12 weeks ( $56 \%$ vs $39 \%$, respectively; $P=0.0026)$. The odds ratio for complete healing for a BLCC-treated ulcer compared with a control-treated ulcer was 2.14 (95\% confidence interval 1.23-3.74). BLCC also accelerated wound closure. Based on Kaplan-Meier estimates, the median time to complete wound closure was 65 days with BLCC compared to 90 days in the control group ( $P=0.0026)$. In the study, patients in the treatment group could have BLCC reapplied at weekly intervals following the initial application, for up to five applications. The average number of BLCC applications was 3.9 per patient (range one to five); $9 \%$ of patients required one application, $10 \%$ two applications, $13 \%$ three applications, $15 \%$ four applications, and $53 \%$ five applications. Ulcer-recurrence rates were similar for the two treatments at 6 months $(5.9 \%$ and $12.9 \%$, respectively; not significant). The rate of adverse reactions was similar in both groups. However, osteomyelitis and lower-limb amputations were less frequent in the BLCC-treated group after 6 months.

Similar results were observed in an international multicenter, randomized, controlled trial. ${ }^{44}$ To be eligible for the study, patients had to have type 1 or type 2 diabetes, age 18-80 years, adequate glycemic control, and a full-thickness neuropathic ulcer for at least 2 weeks prior to the initial screening visit. Eligible patients were randomized to treatment with BLCC in combination with standard care or standard-care therapy alone. Standard care was consistent with international treatment guidelines, and included sharp debridement, salinemoistened dressings, and off-loading. The primary efficacy end point was the time to complete wound healing, defined as full epithelialization with no drainage. Additional efficacy end points included the incidence of complete healing by 12 weeks and ulcer recurrence at 12 weeks after complete healing. The study included a total of 72 patients who comprised the intent-to-treat population: 33 received treatment with BLCC plus standard care therapy, and 39 received standard care alone. Baseline demographics, duration of diabetes, and duration of target ulcer were similar between treatment groups. On average, patients treated with BLCC received 1.8 applications during the course of the study: 13 of 33 patients required only one application, 15 received two applications, and five received three applications). The mean number of 
debridements throughout the 12-week treatment period was 7.2 in the BLCC group and 8.6 in the control group, although this was not statistically significant $(P=0.168)$. Kaplan-Meier curves showed a trend for shorter time to complete healing in the BLCC group compared with the standard-care therapy group ( $P=0.059$, log-rank test). The median time to healing was 84 days with BLCC. This data point could not be determined for the standard-care group, because less than $50 \%$ of the patients in this treatment arm healed. At 12 weeks, the incidence of complete wound closure was 51.5\% (17 of 33) with BLCC and $26.3 \%$ (ten of 38 ) with standard-care therapy alone $(P=0.049)$.

It is interesting to note that in both trials, despite BLCC therapy, up to half of patients did not achieve complete wound closure. In the pivotal US trial, patients could receive up to five applications of BLCC over the course of the 12-week study; the mean number of applications was 3.9. Similarly, in the international trial, the study course was 12 weeks, and the mean number of applications was 1.8 during this time frame. It is therefore unknown whether additional patients would have achieved complete wound closure had the study continued beyond week 12 and/or if patients received additional applications of BLCC. There are multiple factors contributing to nonhealing DFUs, and for those patients not adequately responding to advanced treatments, such as BLCC, clinicians should reevaluate and correct any issues that may be impairing progress (eg, excessive bioburden/infection, inadequate perfusion, poor glycemic control).

\section{Comparative-effectiveness analysis}

BLCC has also been evaluated in a real-world comparativeeffectiveness analysis of patients with DFUs. In this analysis, BLCC was compared with a dehydrated human amnionchorion membrane (dHACM; Epifix, MiMedx Group Inc, Marietta, GA, USA) allograft, a collagenous membrane that contains no living cells. ${ }^{45}$ Data were obtained from a wound care-specific electronic medical record database (Net Health; Pittsburgh, PA, USA) that included patients with DFUs receiving treatment in 2014 at 99 wound-care centers. The analysis considered DFUs between 1 and $25 \mathrm{~cm}^{2}$ of $\leq 1$-year duration and $\leq 20 \%$ area reduction in the 2 weeks prior to the first treatment. This included 155 patients with 163 wounds in the BLCC-treatment group and 63 patients with 63 wounds in the dHACM-treatment group. Baseline wound characteristics were similar, including mean wound sizes of 6 and $5.2 \mathrm{~cm}^{2}$, and mean wound durations of 4.4 and 4.6 months for BLCC and AHACM, respectively. However, the number of applications was statistically significantly different between the treatment groups. Wounds treated with BLCC received on average 2.5 applications, whereas wounds treated with $\mathrm{dHACM}$ allografts received on average 3.5 applications $(P=0.02)$. The estimated incidence of wound closure for BLCC was significantly improved compared with dHACM at 12 weeks (48\% vs $28 \%$ ) and 24 weeks (72\% vs $47 \%)(P=0.01)$. In addition, BLCC treatment significantly improved the median time to DFU wound closure by $49 \%$ (13.3 weeks vs 26 weeks, $P=0.01$ ).

Importantly, results of this comparative-effectiveness analysis were reasonably consistent with those observed in the randomized controlled clinical trials described earlier, and support the efficacy of BLCC in healing wounds in routine clinical practice. ${ }^{45}$ Although the analysis was not designed to assess costs related to wound-care therapy and outcomes, results may suggest that the faster time to wound healing, higher incidence of wound healing, and lower number of applications with BLCC versus the comparator may have potential cost-saving implications. A recent economic analysis assessed the real-world medical resource utilization and costs of DFUs, comparing BLCC against standard-care therapy. ${ }^{46}$ Using Medicare administrative claim data, 502 matched BLCC-standard care patient pairs (matched based on baseline demographics, wound severity, and physician-experience measures) were analyzed for an 18-month period. Compared with their matched standard-care therapy counterparts, BLCC-treated patients incurred more cost for outpatient and physician office visits $(\$ 7,100, P<0.01)$; however, these increased costs were offset by reductions in lower-limb amputations and other resource use, particularly inpatient services. BLCC-treated patients had a $28 \%$ reduction in lower-limb amputations $(11.8 \%$ vs $16.3 \%$ with standard care, $P=0.04), 32 \%$ fewer emergency department visits (4.4 visits vs 6.5 visits, $P<0.0001$ ), and $33 \%$ fewer days hospitalized (10.6 days vs 15.9 days, $P<0.0001)$. Overall, during the course of the 18-month follow-up, BLCC was associated with a $\$ 5,253$ reduction in average per-patient health care cost, suggesting that the use of advanced wound therapy with BLCC may decrease DFU-associated complications and lower overall medical costs through reduced utilization of health care services.

\section{Case studies}

\section{DFU with necrosis}

A 52-year-old man with type 2 diabetes on hemodialysis with lower-extremity peripheral arterial disease presented with a right-foot necrotic ulcer present for 216 days (Figure 4A). The fifth metatarsal was exposed with bone infection. Initial treatment consisted of right-leg arthrectomy revascularization, 


\section{A}

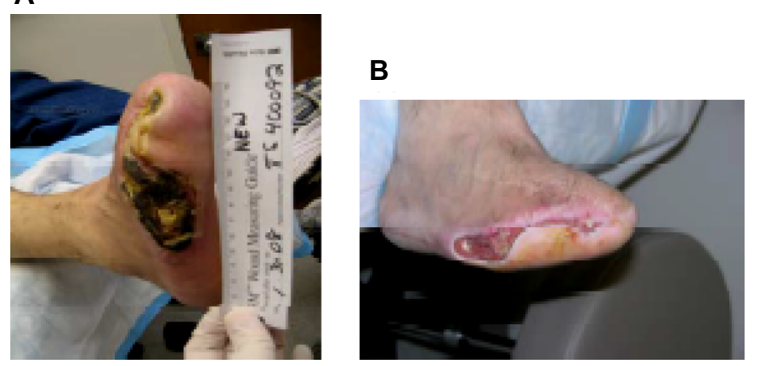

C

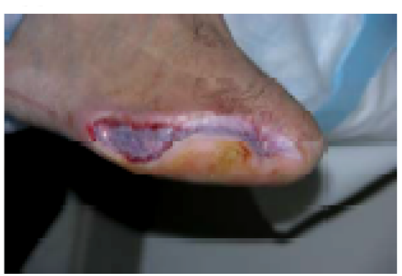

D

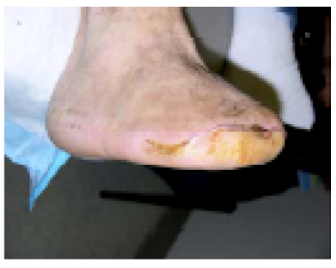

Figure 4 A 52-year-old man with type 2 diabetes.

Notes: (A) Right-foot necrotic ulcer present for 216 days; (B) following standard wound care for 3 months; (C) following initial BLCC application; (D) following three applications of BLCC. This diabetic ulcer was healed at $4 \mathrm{I}$ days.

Abbreviation: BLCC, bilayered living cellular construct.

A

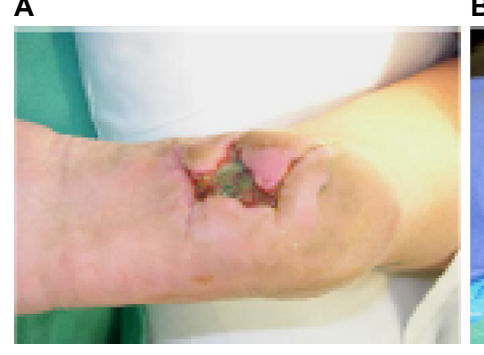

D

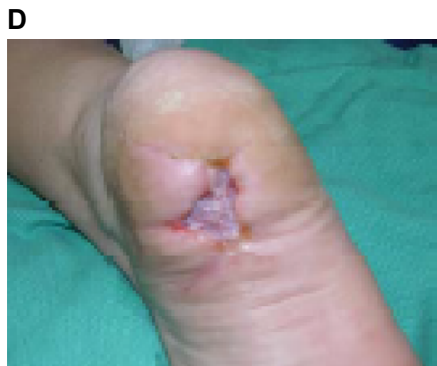

B

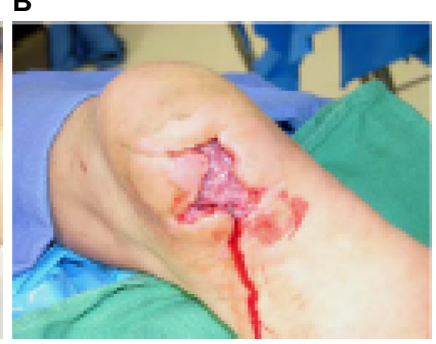

c

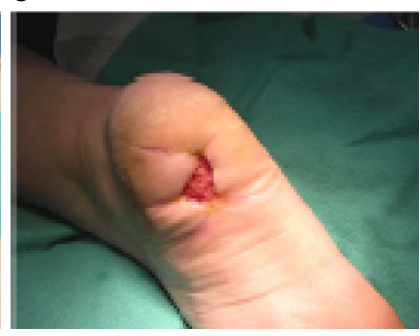

E

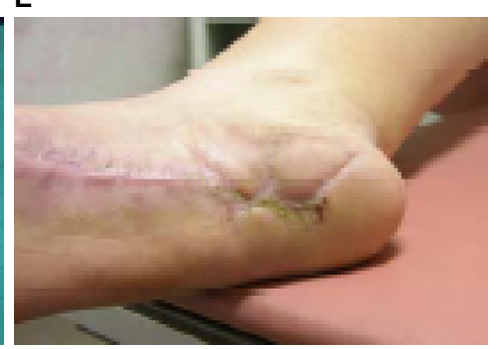

Figure 5 A 39-year-old woman with type 2 diabetes.

Notes: (A) Surgical incision dehiscence; (B) at initial application of BLCC; (C) application of BLCC at day 21; (D) final application of BLCC at day 58; (E) the diabetic ulcer was healed at day 80 .

Abbreviation: BLCC, bilayered living cellular construct.

intravenous antibiotics, partial fifth-metatarsal resection, and extensive ulcer debridement. Wound care after the foot surgery consisted of weekly ulcer sharp debridement and daily papain/ urea/chlorophyllin green ointment for 3 months with wound improvement (Figure 4B). After this point, there was no further wound improvement for 1 month using papain/urea/chlorophyllin green ointment. Following revascularization, BLCC was utilized (Figure 4C). After a total of three applications, there was complete healing in 41 days without reoccurrence (Figure 4D). Patient written informed consent was obtained for publication of this case, as well as the use of any patient images.

\section{Diabetic heel ulcer after a dehisced surgical incision}

A 39-year-old woman with type 2 diabetes had a benign soft-tissue mass excised by a separate surgeon at the plantar aspect of the heel. The incision progressed to complete incision dehiscence (Figure 5A). The patient had a 15-pack-year smoking history and continued to smoke. Wound-care management consisting of topical antibiotics and dry sterile dressing changes with crutch pressure offloading was attempted first for 35 days without improvement in ulcer size. Wound-bed site preparation with initial application of BLCC was initiated, and there was significant ulcer improvement; see photos of initial (Figure 5B) and subsequent application at day 21 (Figure 5C) of BLCC. Following the final BLCC application at day 58 (Figure 5D), the diabetic ulcer was healed at day 80 (Figure 5E). There was no diabetic ulcer reoccurrence at the patient's 2-year follow-up. Patient consent was obtained for publication of this case, as well as the use of any patient images. 


\section{Summary}

Wound healing is a complex process involving a sequence of overlapping but distinct events, including inflammation, proliferation, and remodeling. For proper wound healing, these events must occur with the coordinated efforts of a complex network of cells, their cellular messengers (ie, cytokines and growth factors), and interaction with the ECM. Patients with diabetes have a number of underlying clinical and pathophysiological defects that impair their immune function, making DFUs readily chronic in nature. The goal of treatment is to convert the chronic wound environment to an acute healing environment. However, only a minority of DFUs heal with standard wound care alone. Most DFUs remain "stalled" in one of the phases of the wound-healing process. Cytokines and growth factors, which are readily present in the wound bed of acute wounds, have been found to be insufficiently present or impaired in chronic nonhealing wounds. Moreover, keratinocytes and fibroblasts, which are critical for reepithelialization of the wound and remodeling of the ECM, are often morphologically and functionally abnormal in chronic wounds.

For chronic nonhealing DFUs, the use of advanced therapy with BLCC is a treatment option. BLCC is a bioengineered living-cell-based technology that is FDAapproved for the treatment of DFUs of greater than 3-week duration. It is a bilayered construct containing living keratinocytes (including keratinocyte stem cells) and fibroblasts in an ECM of collagen and other ECM proteins. The presence of both keratinocytes and fibroblasts in BLCC has been shown to facilitate the delivery of a broad array of cytokines and growth factors often deficient in a chronic wound. A recent study found that BLCC reversed patient wounds from a chronic profile to an acute profile. ${ }^{42}$ BLCC has an important body of evidence to support its use in DFUs. Randomized controlled trials demonstrate a greater frequency of complete healing and faster time to healing compared with standard-care therapy alone. ${ }^{43,44} \mathrm{~A}$ comparative-effectiveness analysis showed greater healing rates and faster time to healing of BLCC compared with an active comparator, and supported its use in the realworld clinical setting. ${ }^{45}$ An economic analysis suggests that BLCC may decrease DFU-associated complications and lower overall medical costs through reduced utilization of health care services. ${ }^{46}$

\section{Disclosure}

The author reports that he has served as a consultant with Organogenesis Inc in the past, as well as other orthopedic and wound-care companies. At the time of the manuscript development and submission, the author did not have an existing consulting agreement with Organogenesis Inc. The author reports no other conflicts of interest in this work.

\section{References}

1. Medicines New Zealand. New Zealand's Medicines Landscape: 2015 Wellington: Medicines New Zealand; 2015. Available from: http:// www.medicinesnz.co.nz/assets/Documents/New-Zealands-MedicinesLandscape-2017.pdf. Accessed November 14, 2016.

2. Coppell KJ, Mann JI, Williams SM, et al. Prevalence of diagnosed and undiagnosed diabetes and prediabetes in New Zealand: findings from the 2008/09 Adult Nutrition Survey. NZ Med J. 2013;126(1370):23-42.

3. Australian Institute of Health and Welfare. Diabetes: Australian Facts 2008. Canberra: AIHW; 2008. Available from: http://www.aihw.gov.au/ WorkArea/DownloadAsset.aspx?id=6442454991. Accessed August 18, 2016.

4. Lazzarini PA, Gurr JM, Rogers JR, Schox A, Bergin SM. Diabetes foot disease: the Cinderella of Australian diabetes management? J Foot Ankle Res. 2012;5(1):24

5. Yazdanpanah L, Nasiri M, Adarvishi S. Literature review on the management of diabetic foot ulcer. World J Diabetes. 2015;15:6(1) 37-53.

6. Reiber GE. The epidemiology of diabetic foot problems. Diabet Med 1996;13 Suppl 1:S6-S11.

7. Reiber GE, Boyko EJ, Smith DG. Lower extremity foot ulcers and amputations in diabetes. In: Harris MI, Cowie CC, Stern MP, Boydo EJ, Reiber GE, Bennett PH, editors. Diabetes in America. 2nd ed. Washington: Department of Health and Human Services; 1995.

8. Carpenter S, Davis S, Fitzgerald R, et al. Expert recommendations for optimizing outcomes in the management of biofilm to promote healing of chronic wounds. Wounds. 2016;28(6 Suppl):S1-S20.

9. Gurtner GC, Werner S, Barrandon Y, Longaker MT. Wound repair and regeneration. Nature. 2008;453(7193):314-321.

10. Kirsner RS, Eaglstein WH. The wound healing process. Dermatol Clin. 1993;11(4):629-640.

11. Lobmann R, Schultz G, Lehnert H. Proteases and the diabetic foot syndrome: mechanisms and therapeutic implications. Diabetes Care. 2005;28(2):461-471.

12. Hunt TK, Hopf H, Hussain Z. Physiology of wound healing. Adv Skin Wound Care. 2000;13(2 Suppl):6-11.

13. Frykberg RG, Banks J. Challenges in the treatment of chronic wounds. Adv Wound Care. 2015;4(9):560-582.

14. Kane D, Krasner D, editors. Chronic Wound Care: A Clinical Source Book for Healthcare Professionals. 2nd ed. Wayne (PA): Health Management Publications; 1997.

15. Schultz GS, Sibbald RG, Falanga V, et al. Wound bed preparation: a systematic approach to wound management. Wound Rep Reg. 2003;11 Suppl 1:S1-S28.

16. Tsourdi E, Barthel A, Rietzsch H, Reichel A, Bornstein SR. Current aspects in the pathophysiology and treatment of chronic wounds in diabetes mellitus. Biomed Res Int. 2013;2013:385641.

17. Kavitha KV, Tiwari S, Purandare VB, Khedkar S, Bhosale SS, Unnikrishnan AG. Choice of wound care in diabetic foot ulcer: a practical approach. World J Diabetes. 2014;5(4):546-556.

18. Marhoffer W, Stein M, Maeser E, Federlin K. Impairment of polymorphonuclear leukocyte function and metabolic control of diabetes. Diabetes Care. 1992;15(2):256-260.

19. Association for the Advancement of Wound Care. Advancing Your Practice: Understanding Wound Infection and the Role of Biofilms. Malvern (PA): AAWC; 2008.

20. Phillips P, Sampson E, Yang Q, Antonelli P, Progulske-Fox A, Schultz G. Bacterial biofilms in wounds. Wound Heal S Afr. 2008;1(2):10-12.

21. James GA, Swogger E, Wolcott R, et al. Biofilms in chronic wounds Wound Repair Regen. 2008;16(1):37-44. 
22. Yang Q, Phillips PL, Sampson E, et al. Development of a novel in vitro porcine skin explant model for the assessment of mature bacterial biofilms. Wound Repair Regen. 2013;21(5):704-714.

23. Zhao G, Usui ML, Lippman SI, et al. Biofilms and inflammation in chronic wounds. Adv Wound Care (New Rochelle). 2013;2(7): 389-399.

24. Metcalf DG, Bowler PG. Biofilm delays wound healing: a review of the evidence. Burns Trauma. 2013;(1):5-12.

25. Barrientos S, Stojadinovic O, Golinko MS, Brem H, Tomic-Canic M. Growth factors and cytokines in wound healing. Wound Repair Regen. 2008;16(5):585-601.

26. Cook H, Davies KJ, Harding KG, Thomas DW. Defective extracellular matrix reorganization by chronic wound fibroblasts is associated with alterations in TIMP-1, TIMP-2, and MMP-2 activity. J Invest Dermatol. 2000;115(2):225-233.

27. Falanga V. Wound healing and its impairment in the diabetic foot. Lancet. 2005;366(9498):1736-1743.

28. Lavery LA, Davis KE, Berriman SJ, et al. WHS guidelines update: diabetic foot ulcer treatment guidelines. Wound Repair Regen. 2016;24(1): 112-126.

29. Margolis DJ, Kantor J, Berlin JA. Healing of diabetic neuropathic foot ulcers receiving standard treatment: a meta-analysis. Diabetes Care. 1999;22(5):692-695.

30. Coerper S, Beckert S, Küper MA, Jekov M, Königsrainer A. Fifty percent area reduction after 4 weeks of treatment is a reliable indicator for healing: analysis of a single-center cohort of 704 diabetic patients. J Diabetes Complications. 2009;23(1):49-53.

31. Margolis DJ, Gelfand JM, Hoffstad O, Berlin JA. Surrogate end points for the treatment of diabetic neuropathic foot ulcers. Diabetes Care. 2003;26(6):1696-1700.

32. Sheehan P, Jones P, Casselli A, Giurini JM, Veves A. Percent change in wound area of diabetic foot ulcers over a 4-week period is a robust predictor of complete healing in a 12 -week prospective trial. Diabetes Care. 2003;26(6):1879-1882.

33. Margolis DJ, Kantor J, Santanna J, Strom BL, Berlin JA. Risk factors for delayed healing of neuropathic diabetic foot ulcers: a pooled analysis. Arch Dermatol. 2000;136(12):1531-1535.
34. Brem H, Young J, Tomic-Canic M, Isaacs C, Ehrlich HP. Clinical efficacy and mechanism of bilayered living human skin equivalent (HSE) in treatment of diabetic foot ulcers. Surg Technol Int. 2003;11:23-31.

35. Kirsner RS, Warriner R, Michela M. Advanced biological therapies for diabetic foot ulcers. Arch Dermatol. 2010;146(8):857-862.

36. Carlson M, Faria K, Shamis Y, Leman J, Ronfard V, Garlick J. Epidermal stem cells are preserved during commercial-scale manufacture of a bilayered, living cellular construct (Apligraf). Tissue Eng Part A. 2011;17(3-4):487-493.

37. Hardin-Young J, Parenteau NL. Bilayered skin constructs. In: Atala A, Lanza RP, editors. Methods of Tissue Engineering. San Diego: Academic Press; 2002:1177-1188.

38. Osborne CS, Schmid P. Epidermal-dermal interactions regulate gelatinase activity in Apligraf, a tissue-engineered human skin equivalent. Br J Dermatol. 2002;146(1):26-31.

39. Badiavas EV, Paquette D, Carson P, Falanga V. Human chronic wounds treated with bioengineered skin: histologic evidence of host-graft interactions. J Am Acad Dermatol. 2002;46(4):524-530.

40. Griffiths M, Ojeh N, Livingstone R, Price R, Navsaria H. Survival of Apligraf in acute human wounds. Tissue Eng. 2004;10(7-8):1180-1195.

41. Stojanovic O, Ramirez H, Patel S, et al. Genomic insight into molecular mechanisms of action of bilayered living cellular construct in nonhealing venous leg ulcers. Wound Repair Regen. 2014;22(2):A60.

42. Veves A, Falanga V, Armstrong DG, Sabolinski ML. Graftskin, a human skin equivalent, is effective in the management of noninfected neuropathic diabetic foot ulcers: a prospective randomized multicenter clinical trial. Diabetes Care. 2001;24(2):290-295.

43. Edmonds M. Apligraf in the treatment of neuropathic foot ulcers. Int $J$ Low Extrem Wounds. 2009;8(1):11-18.

44. Kirsner RS, Sabolinski ML, Parsons NB, Skornicki M, Marston WA. Comparative effectiveness of a bioengineered living cellular construct vs. a dehydrated human amniotic membrane allograft for the treatment of diabetic foot ulcers in a real world setting. Wound Repair Regen. 2015; 23(5):737-744.

45. Rice JB, Desai U, Ristovska L, et al. Economic outcomes among Medicare patients receiving bioengineered cellular technologies for treatment of diabetic foot ulcers. J Med Econ. 2015;18(8):586-595.
Chronic Wound Care Management and Research

\section{Publish your work in this journal}

Chronic Wound Care Management and Research is an international, peer reviewed, open access, online journal publishing original research, reviews, editorials, and commentaries on the causes and management of chronic wounds and the major issues related to chronic wound management. Topics also include chronic wounds as comorbidities to other

\section{Dovepress}

conditions, patient adherence to therapy, and the economic burden of chronic wounds. The manuscript management system is completely online and includes a very quick and fair peer review system, which is all easy to use. Visit http://www.dovepress.com/testimonials.php to read real quotes from published authors. 